\section{Feasibility of Doppler variance imaging for red blood cell aggregation characterization}

\author{
Xiangqun $\mathrm{Xu}^{\mathrm{a}, *}$ Yeh-Chan Ahn, ${ }^{\mathrm{b}}$ and Zhongping Chen ${ }^{\mathrm{b}}$ \\ ${ }^{a}$ Zhejiang Sci-Tech University, School of Science, Higher \\ Education park in Xiasha, Hangzhou, Zhejiang \\ 310018, China \\ ${ }^{b}$ University of California, Beckman Laser Institute, 1000 \\ Health Sciences Road, Irvine, California 92612
}

\begin{abstract}
An abnormal level of red blood cell (RBC) aggregation is a clinical condition associated with many pathologies. Our purpose is to investigate the feasibility of spectral domain phase-resolved optical Doppler tomography incorporated with Doppler variance imaging to characterize RBC aggregation in flowing blood. Variance/ standard deviation (SD) of the Doppler frequency spectrum of porcine blood flowing through a glass tube (diameter $300 \mu \mathrm{m}$ ) at $4.7 \mathrm{~mm} / \mathrm{s}$ is measured. For nonaggregating RBCs with hematocrits of 20,40 , and $60 \%$, the mean centerline SD values were $34.2 \pm 1.2,47.8 \pm 1.0$, and $47.2 \pm 0.5$, respectively. After dextran 500 induction, the SD value decreased significantly to $29.9 \pm 0.7$, $37.9 \pm 3.0$, and $40.1 \pm 0.6(P<0.01)$ in the aggregated blood, respectively. The results demonstrate that Doppler variance imaging using the SD value is capable of differentiating normal and aggregated blood. ๑ 2009 Society of Photo-Optical Instrumentation Engineers. [DOI: 10.1117/1.3275464]
\end{abstract}

Keywords: Doppler variance image; red blood cell aggregation; optical Doppler tomography; velocity variation.

Paper 09274LR received Jun. 30, 2009; revised manuscript received Aug. 22, 2009; accepted for publication Oct. 28, 2009; published online Dec. 21, 2009

Red blood cell (RBC) aggregation is an important physiological phenomenon responsible for the non-Newtonian behavior of blood. RBC aggregation is normally present in humans and in many other "athletic" species. An abnormally high level of RBC aggregation has long been considered to be principally of pathophysiologic importance since aggregation is elevated in many disease states. Understanding RBC aggregation could bring new insights concerning these pathologies.

Optical coherence tomography (OCT)/Doppler OCT (ODT) is able to achieve high imaging speed, high velocity sensitivity, and high spatial resolution simultaneously. OCT/ ODT has been used to study blood optical clearing, aggregation, sedimentation, and hematocrit (HCT) in our and other groups. ${ }^{1-7}$ In our previous work, we demonstrated that the slope of the OCT depth-reflectivity profile decreased with the level of RBC aggregation. ${ }^{6,7}$ This is so because RBC aggregation leads ${ }^{8}$ to a decrease in scattering of RBCs. Unfortunately, the relationship between the slope and the aggregation of RBCs could not be directly used for in vivo determination

*Address all correspondence to: Xiangqun Xu. Tel: 86-137-7783-3176; Fax: 86571-8770-2220; E-mail: xuxiangqun@zstu.edu.cn

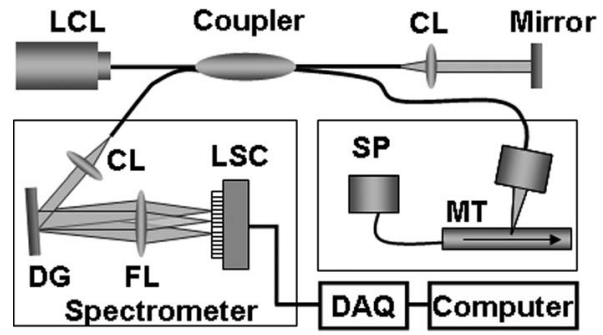

Fig. 1 Schematic diagram of the SDODT. Incident light was inclined by 5 deg from the vertical. CL, collimating lens; DG, diffraction grating; FL; focusing lens; LCL, low coherent light; LSC, line scan camera; MT, microtube; SP, syringe pump; DAQ, data acquisition.

of RBC aggregation. The scattering properties of vessels, surrounding tissue, and HCT would affect the slope of the OCT depth-reflectivity profile. A new principle for blood aggregation characterization is worth studying except for measuring scattering by OCT. We recently demonstrated spectral domain ODT (SDODT) for measuring blood flow velocity under various circumstances. ${ }^{9}$ Doppler variance tomography using the variance of the Doppler frequency spectrum to more efficiently map the flows in highly scattering biological tissues has been reported. ${ }^{10,11}$ In addition to the local velocity information, phase-resolved ODT systems give the variance of the Doppler frequency spectrum, ${ }^{10,11}$ which is given by

$$
\sigma^{2}=\frac{2}{T^{2}}\left\{1-\frac{\left|(1 / N) \sum_{j=1}^{N}\left[f_{j T}(z) f_{(j+1) T}^{*}(z)\right]\right|}{(1 / N) \sum_{j=1}^{N}\left[f_{j T}(z) \cdot f_{j T}^{*}(z)\right]}\right\},
$$

where $N$ is the number of A-lines used for averaging; $T$ is the time interval between two exposures, and $f(z)$ is a complex signal from an inverse Fourier transform of the interference pattern. The corresponding Doppler shift can be estimated by comparing the phasors of $f(z)$ between two complex signals during two exposures at a same location. The phase change is recorded by the product of $f_{j T}(z)$ and $f_{(j+1) T}^{*}(z)$, where * denotes the conjugate operation. The value of $\sigma^{2}$ depends on the flow velocity distribution. Variations of flow velocity broaden the Doppler frequency spectrum and result in a larger $\sigma^{2}$. Thus, the Doppler variance obtained by a phase-resolved ODT system can be an indicator of flow variations.

Individual particles within concentrated suspensions exhibit significant random movements when the suspension is subjected to shear flow. These movements are dependent on the local shear rate of the suspension and on particle size. ${ }^{12}$ Previous studies have shown variation in the instantaneous velocity of individual RBCs and the effect of aggregation on the dispersion of RBCs flowing in venules. ${ }^{12,13}$ RBC aggregation in the flow stream decreases the variability in instantaneous cellular velocity. ${ }^{13}$ This effect is most likely the result of an increased particle size. The magnitude of particle movements is decreased when the particle diameter increases. ${ }^{12,13}$

1083-3668/2009/14(6)/060507/3/\$25.00 @ 2009 SPIE 


\section{JBO LETTERS}

In this paper, we investigate the feasibility of phase-resolved SDODT combined with the Doppler variance image to characterize RBC aggregation in flowing blood.

Figure 1 is a schematic of the SDOCT system. A superluminescent diode with a spectrum centered at $1310 \mathrm{~nm}$, a full width at half maximum bandwidth of $95 \mathrm{~nm}$, and a total delivered power of $8 \mathrm{~mW}$ was used. Back-reflected light from the reference and sample arms was guided into a spectrometer and dispersed over a $1 \times 1024 \mathrm{InGaAs}$ detector array at $7.7 \mathrm{kHz}$ (corresponding to $1 / T$ ). The total wavelength range spreading on the detector array was $130 \mathrm{~nm}$, corresponding to a spectral resolution of $0.13 \mathrm{~nm}$ and an imaging depth of $3.6 \mathrm{~mm}$ in air. The resulting axial resolution was about $8 \mu \mathrm{m}$, and the lateral resolution of the system was ${ }^{6,9}$ about $13.8 \mu \mathrm{m}$.

In the phase-resolved OCT/ODT, the phase change of the interference fringe between sequential A-line scans was used $^{10}$ to calculate the Doppler-frequency shift. The interference signal was inversely Fourier transformed after reducing the dc component. Cross-correlations between sequential A-scans are taken. ${ }^{11}$ The standard deviation (SD) of phase shift between two consecutive exposures $(=\sigma T)$ was calculated from the cross-correlation using Eq. (1).

Various HCT levels were obtained by mixing extracted RBCs with porcine plasma using ethylenediamine tetraacetic acid (EDTA) as an anticoagulant (Animal Technologies, Inc., Tyler, Texas). Different suspensions of normal RBCs, artificially aggregated with dextran, were prepared. RBCs were resuspended in plasma of dextran 500 (Dx500, average molecular mass $500 \mathrm{kDa}$; Sigma, United State) at HCTs of 20, 40 , and $60 \%$. A plasma dextran concentration of $2 \%$ in weight/volume (w/v) was used to induce moderate RBC aggregation. All OCT measurements were performed just after RBC suspensions were prepared and pumped through a round glass capillary tube with a 300- $\mu \mathrm{m}$ inner diameter (VitroCom Inc., Mountain Lakes, New Jersey) at a Doppler angle of 5 deg. A programmable syringe pump (Harvard Apparatus, Holliston, Massachusetts) was used to make the blood flow with an approximately constant speed at a rate of $20 \mu \mathrm{l} / \mathrm{min}$, corresponding to an average flow velocity of $4.7 \mathrm{~mm} / \mathrm{s}$ within the glass capillary. The flow rate simulates the real values within major human retinal vessels with diameters $>200 \mu \mathrm{m}$. OCT "M-scans" (a single A-line repeatedly acquired in time without transverse scanning) were performed. One thousand and twenty-four OCT A-scans were averaged for each data set. Triplicate samples were prepared and measured for each HCT. All final results of SD values for both normal and aggregated blood were means of triplicate experiments. A paired $t$ test statistical analysis was applied for the differences in SD between the control group (without dextran) and the treated group (with dextran). For all tests, a level of 0.05 was chosen as a significant level.

Figure 2 shows the Doppler variance images that give the variation of the blood (HCT $=40 \%)$ velocity distribution for the blood without dextran [Fig. 2(a)] and the aggregated blood with $2 \%$ Dx500 [Fig. 2(b)] at HCT of $40 \%$ flowing through a $300-\mu \mathrm{m}$ glass tube at a flow rate of $4.7 \mathrm{~mm} / \mathrm{s}$. The presence of different velocity variations between the control and the aggregated within the glass tube was observed.

The quantitative values of SD along the depth calculated according to Eq. (1) for the control and the treated blood were determined and are plotted in Fig. 3 for $\mathrm{HCT}=40 \%$ [Fig.

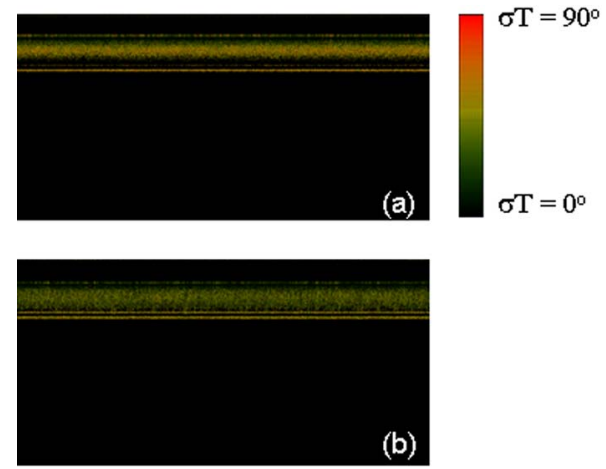

Fig. 2 Doppler variance images of the blood at HCT=40\% for (a) normal and (b) dextran-treated blood. The axial axis represents scan depth $(0$ to $3.6 \mathrm{~mm})$ and the horizontal axis represents 2000 A-lines.

3(a)] and HCT $=60 \%$ [Fig. 3(b)]. For the control blood at $\mathrm{HCT}=40$ or $60 \%$, the upper lines of the SD profiles in Figs. $3(\mathrm{a})$ and 3(b) show that the velocity variations vary with radial position in the glass tube. A higher SD is found near the center of the tube where the shear rate is minimal for the flow. There is asymmetry in the SD profiles along the pixel depth. The SD in the bottom of the tube is higher than that in the top of the tube. It is possible that the increase in RBC density in the unit area associated with RBC sedimentation in this in vitro model, leading to an increase in random collision among RBCs and thus velocity variations. From the lower lines of the SD profiles in Figs. 3(a) and 3(b) for the aggregated blood, we can see the motion of the particles is strongly influenced by the RBC aggregation. The decrease in the SD in the center of the tube could be the result of a decrease in flow variations due to changes in the size of scatterers associated with RBC aggregation. When aggregates migrate to the center of the tube, particle size will be greater in the center of the tube than near the tube wall and effective viscosity will be greater there as well. ${ }^{12}$

The mean values of $47.8 \pm 0.99$ and $47.2 \pm 0.45$ for the control group at HCTs of 40 and $60 \%$, respectively, have no significant differences. The value of $37.9 \pm 3.06$ for dextrantreated blood with HCT of $40 \%$ is not significantly different from that of $40.1 \pm 0.55$ for the blood with HCT of $60 \%$ either. However, both of them are significantly $(P<0.01)$ lower than the values for the corresponding control blood at the two HCTs. The addition of dextran decreases the SD by 20.7 and $15.0 \%$ for HCTs of 40 and $60 \%$, respectively. This finding is consistent with the in vivo work ${ }^{13}$ that the magnitude of the velocity variations in dextran-treated blood is less than that in nonaggregating blood. It is most likely that RBCs residing in aggregates could be both shielded from interactions with surrounding cells and also hindered in their movements or the magnitude of particle movements is decreased when the particle diameter increases. ${ }^{13}$ Note that the addition of dextran would increase the blood viscosity. Although we do not know much the increased viscosity with dextran contributes to the decreased velocity variations in dextran-treated blood, nevertheless, the variance imaging can differentiate the velocity variation change between the two groups. Interestingly, the SD in normal blood or aggregated blood is not dependent on HCT when HCT around or greater than $40 \%$ (normal HCT in a human female, $45 \%$ in a human male). 

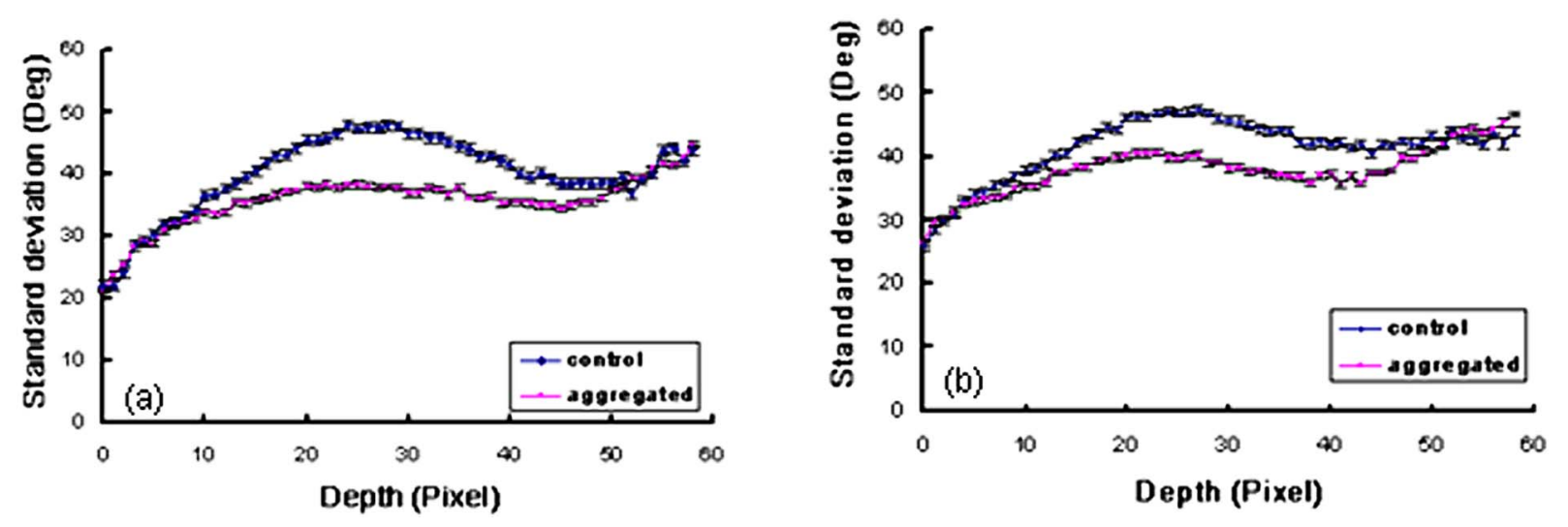

Fig. 3 SD versus depth profiles for normal and dextran-treated blood at HCTs, of (a) $40 \%$ and (b) $60 \%$.

The centerline SD of $34.2 \pm 1.16$ for the normal blood at HCT of $20 \%$ is much lower than that at 40 or $60 \%$. It is possible that the numbers of RBCs in a unit area will decrease according to the decrease in the concentration of HCT. This causes a decrease in random collision among RBCs and thus velocity variations. The value for dextran-treated blood $(29.9 \pm 0.66)$ is significantly $(P<0.01)$ lower than the value for the control. The SD is decreased by $14.6 \%$ at blood aggregation. The results demonstrate that Doppler variance imaging using the SD value is capable of differentiating normal and aggregated blood at HCTs from 20 to $60 \%$.

A method to determine blood flow, HCT, and blood cell aggregation in a noninvasive manner will be important for many clinical applications. Our previous results demonstrated a significant underestimation of HCT using the slope of the OCT depth reflectivity profile caused by RBC aggregation. Compensation for the discrepancy caused by RBC aggregation is necessary to develop a novel instrument based on OCT for in vivo measurements ${ }^{7}$ of the real value of blood HCT. In addition, we reported the relationship between RBC aggregation and the slope of the OCT depth reflectivity profile, however, the latter can not be used to measure RBC aggregation either because the slope is also dependent ${ }^{6,7}$ on HCT. Therefore, developing various OCT methods except for scattering measurement and calibration methods that enables simultaneous measurements of RBC aggregation and HCT are essential for accurate determination of HCT and aggregation. The preceding results demonstrated that the SD value using Doppler variance imaging is not dependent on normal HCT. With the slope measurement and calibration methods, the change in $\mathrm{SD}$ value also has potential to monitor RBC aggregation for a lower HCT. To overcome the OCT penetration depth limitation in scattering tissues, SDOCT/ODT combined with retinal tracking to target retinal vessels would be a solution for in vivo blood rheology measurements. Retina veins would be a better target that exhibits a lesser effect of the elasticity of the vessels and the pulsatile nature of the blood flow. Alternatively, a microfluidic system could be developed for an ex vivo point-of-care test.

In this paper, the feasibility of phase-resolved ODT/ Doppler variance imaging to characterize the level of blood aggregation was preliminarily evaluated. Studies on the spa- tial and temporal attributes of RBC aggregation as a function of shear rate, HCT, extensional flows, and in vivo measurement combined with retinal tracking are underway.

\section{Acknowledgments}

This work was supported by research grants from the National Natural Science Foundation of China (Grant No. 30870675), the National Institutes of Health (Grants EB-00293, CA91717, and RR-01192), the Air Force Office of Scientific Research (Grant No. FA9550-0401-0101), and the Beckman Laser Institute Endowment.

\section{References}

1. Z. Chen, "Noninvasive imaging of in vivo blood flow velocity using optical Doppler tomography," Opt. Lett. 22, 1119-1121 (1997).

2. M. Brezinski, "Index matching to improve OCT imaging through blood," Circulation 103, 1999-2003 (2001).

3. V. V. Tuchin, "Dynamic optical coherence tomography in optical clear, sedimentation and aggregation study of immersed blood," Appl. Opt. 41, 258-271 (2002).

4. X. Xu, "Effect of dextran-induced changes in refractive index and aggregation on optical properties of whole blood," Phys. Med. Biol. 48, 1205-1221 (2003).

5. Y. Kirillin, "Effect of red blood cell aggregation and sedimentation on optical coherence tomography signals from blood samples," $J$. Phys. D: Appl. Phys. 38, 2582-2598 (2005).

6. X. Xu, "Optical clearing of flowing blood using dextrans with spectral domain optical coherence tomography," J. Biomed. Opt. 13(2), 021107-1-6 (2008)

7. X. Xu, "Effect of erythrocyte aggregation on hematocrit measurement using spectral domain optical coherence tomography," IEEE Trans. Biomed. Eng. 55(12), 2753-2758 (2008).

8. A. V. Priezzhev, "Aggregation and disaggregation of erythrocytes in whole blood: study by backscattering technique," J. Biomed. Opt. 4, 76-84 (1999).

9. Y.-C. Ahn, "Optical sectioning for microfluidics: secondary flow and mixing in a meandering microchannel," Lab Chip 8, 125-133 (2008).

10. Y. Zhao, "Doppler standard deviation imaging for clinical monitoring of in vivo human skin blood flow," Opt. Lett. 25(18), 1358-1360 (2000).

11. L. Wang, "Frequency domain phase-resolved optical Doppler and Doppler variance tomography," Opt. Commun. 242, 345-350 (2004).

12. J. J. Bishop, "Rheological effects of red blood cell aggregation in the venous network: a review of recent studies," Biorheology 38, 263274 (2001).

13. J. J. Bishop, "Effect of aggregation and shear rate on the dispersion of red blood cells flowing in venules," Am. J. Physiol. Heart Circ. Physiol. 283, H1985-H1996 (2002). 